\title{
Anesthesia Outside the Operating Room, 2nd Edition
}

\author{
Richard D. Urman, Wendy L. Gross, Beverly K. Philip (Editors). Oxford University Press, \\ 2018, \$165.00, hardcover, 440 pages. ISBN-13: 9780190495756
}

\author{
Kaitlin Duncan, MD (D)
}

Received: 28 November 2018/Accepted: 29 November 2018/Published online: 13 December 2018

(C) Canadian Anesthesiologists' Society 2019

It is undeniably true that we as a specialty are spending more time taking care of sicker patients in more remote settings. A recent analysis published in Anesthesia \& Analgesia found that, in the United States, the proportion of non-operating room anesthesia (NORA) cases increased from $28.3 \%$ to $35.9 \%$ between 2010 and 2014, as did patient age, American Society of Anesthesiologists physical status classification, and the number of cases performed outside of normal working hours. ${ }^{1}$ The relevance of this increasing non-OR work is underscored by the 2018 Anesthesia Closed Claims update showing that complications such as adverse respiratory events and aspiration pneumonitis occur more frequently in NORA cases and that malpractice claims for deaths are more common in non-OR settings than in the OR. ${ }^{2}$ Plainly, we as anesthesiologists need to become comfortable working in these environments. The second edition of Anesthesia Outside the Operating Room from the Oxford University Press is therefore a timely addition to the subject at hand.

Widely published authors with backgrounds in perioperative medicine and ambulatory care, the editors of Anesthesia Outside the Operating Room have succeeded in curating an exhaustive reference book on a rapidly changing field that at times seems challenging. Anesthesia Outside the Operating Room is available in print and as an e-book, allowing inherent portability with a user interface that is easy to navigate. It also offers the option to download individual chapters in portable document format (PDF). This textbook is unusual in that it casts a wide net in terms of its target audience. Although easily appropriate for attending consultant-level anesthesiologists who may be unfamiliar with specific non-OR procedures, the subject

\footnotetext{
K. Duncan, MD ( $\square)$

Faculty of Health Sciences, University of Manitoba, Winnipeg, MB, Canada

e-mail: kait.duncan@gmail.com
}

matter is also pertinent to anesthesia trainees for whom NORA exposure may not be a routine part of their curriculum. In addition, it includes relevant information for non-physician anesthesia providers.

Although NORA is a rapidly evolving field, the 37 chapters and five appendices of this text are impressively comprehensive. The authors begin the text with a bird'seye view of the challenges faced in non-OR settings, excellently summarizing anesthetic techniques, perianesthetic care, and challenges posed by patient monitoring in non-traditional environments. This material is followed by important discussions of the administrative, financial, and quality improvement aspects of NORA which are often overlooked by anesthesia providers but are central to the complexities of providing care in this setting. The chapter structure then narrows to focus specifically on each procedural location, ranging from commonly encountered gastrointestinal and radiologic procedures to the emerging frontiers of interventional pulmonology. For the travel-conscious anesthesiologist, there is even a section that addresses emergency care onboard an aircraft. These chapters provide an extensive overview of nearly any non-OR location imaginable, the procedures that might be needed, and the attendant anesthetic considerations.

This second edition of Anesthesia Outside the Operating Room differs from its original edition in that it adds four new chapters - on financial analysis, anesthesia information management systems (AIMS), radiation safety, and the future of NORA. It also adds two new appendices that address Basic and Advanced Cardiovascular Life Support and pediatric resuscitation. Certain chapters, such as those that address homeland disasters and anesthesia in military settings, have a certain American flavour, but they remain an interesting read and are potentially applicable to a Canadian setting. All in all, the extensive reference lists and inclusion of recent 
American guidelines make this book a highly accessible resource for Canadian anesthesiologists.

Most chapters are written in a narrative style interwoven with supporting evidence and earmarked by clear headings that facilitate rapid information retrieval. The multiplecontributor format begets a degree of repetition throughout the text, however, and the reader might benefit from a more cohesive approach to the structure and formatting among chapters. A summary of key points for each chapter could assist with this approach and might be considered for future editions.

Anesthesia Outside the Operating Room stands up well in comparison to other references on the same subject, in large part because of its extensive coverage of NORA procedures and locations. Compared to other texts, such as Non-Operating Room Anesthesia: Expert Consult and Out of Operating Room Anesthesia: A Comprehensive Review, this reference goes beyond discussion of anesthesia considerations to engage in a more deliberate focus on the technical aspects of each NORA procedure. Although this approach may be considered outside the scope of providing knowledge about anesthetic procedures, the exploration of indications for, and types of, procedures adds significantly to a more global understanding of the anesthesiologist's role in providing NORA.
Overall, this reference book comprehensively presents the anesthetic, procedural, and administrative considerations associated with providing care in non-OR settings in a manner that is both engaging and relevant to a diverse range of anesthesia personnel. Considering the speed at which non-OR procedures are evolving in an environment of increasingly constrained resources, the utility of such a reference in streamlining anesthetic care in both community and tertiary centers alike cannot be overemphasized.

Conflicts of interest None declared.

Editorial responsibility This submission was handled by Dr. Hilary P. Grocott, Editor-in-Chief, Canadian Journal of Anesthesia.

\section{References}

1. Nagrebetsky A, Gabriel RA, Dutton RP, Urman RD. Growth of nonoperating room anesthesia care in the United States: a contemporary trends analysis. Anesth Analg 2017; 124: 1261-7.

2. Woodward ZG, Urman RD, Domino KB. Safety of non-operating room anesthesia: a closed claims update. Anesthesiology Clin 2017; 35: 569-81. 